\title{
Percepciones sobre el Bienestar Docente del Profesorado de EFL en Formación ${ }^{1}$
}

\author{
Manuel Jesús Cardoso Pulido \\ JuAN RAMÓN GUIJARRO OJEDA \\ Universidad de Granada
}

Recibido: 28 abril 2017 / Aceptado: 26 julio 2017

ISSN: $1697-7467$

\begin{abstract}
RESUMEN: Este artículo pretende conocer cuáles son las percepciones de los maestros y maestras de inglés como lengua extranjera (EFL) en materia de bienestar docente, una vez que están realizando sus prácticas externas en un centro escolar. Hemos utilizado una metodología de investigación cualitativa a través de un grupo de discusión integrado por doce estudiantes del Grado en Educación Primaria de la Universidad de Granada. El análisis de los resultados revela que esta cohorte percibe una formación universitaria en bienestar docente y transversal muy deficitaria. Asimismo, se pone de manifiesto que los planes de estudio no recogen supuestos prácticos y que las diferentes materias del plan de estudios no reflejan la realidad que se vive en las aulas; situaciones todas estas que influyen negativamente en su bienestar docente.

Palabras clave: bienestar docente, profesorado de EFL en formación, investigación cualitativa, grupo de discusión.
\end{abstract}

\section{EFL Teacher Trainees' Perceptions on Teacher Wellbeing}

ABSTRACT: This paper pursues to know how EFL teacher trainees perceive teacher wellbeing while they are immersed in their school practice period. The methodology used is based on a qualitative approach, more specifically, a focus group composed of twelve EFL pre-service teachers (University of Granada). The results of the analysis shed light on their lack of training in teacher wellbeing. In addition, there is a claim that the teaching curriculum does not take into account case studies and they also state that the course content of the degree in Primary Education do not fit with the reality of the classroom they are experiencing. These findings clearly reflect a negative impact on their teacher wellbeing.

Keywords: teacher wellbeing, EFL pre-service teachers, qualitative research, focus group

\section{INTRODUCCIÓN}

La justificación de nuestro estudio reside en conocer en profundidad los elementos que

\footnotetext{
${ }^{1}$ Este estudio forma parte del proyecto I+D: Estudio del bienestar docente del profesorado de lenguas ante los retos del plurilingüismo y las aulas multiculturales, financiado por el Ministerio de Cultura, Educación y Deportes (ref. EDU2013-44890-P) y realizado por investigadores de la Universidad de Granada y de la Universidad de Cambridge.
} 
afectan al bienestar docente durante la formación inicial del profesorado de lenguas mientras estos realizan sus prácticas externas. Hemos utilizado herramientas propias de una metodología cualitativa como es el grupo de discusión puesto que nos permite obtener información en un ambiente permisivo, relajado y confortable en el que los participantes exponen sus argumentos e ideas comunes de manera que se influencien mutuamente (Krueger, 1991; Pérez y Ruiz, 2014). Todos los participantes son estudiantes que cursaban la materia de prácticas externas en colegios durante el octavo y último semestre del Grado en Educación Primaria en la Universidad de Granada.

La motivación para realizar esta investigación viene dada por la escasez de referencias que los actuales planes de estudio del Grado en Educación Primaria ofrecen a la formación en bienestar docente. Algunas materias dedican cierto contenido relacionado directa o indirectamente con el bienestar docente como, por ejemplo: la convivencia en el aula (Sociología de la Educación) o el fomento de actitudes positivas hacia el aprendizaje y el uso del modelo de las inteligencias múltiples de Gardner (Enseñanza y Aprendizaje de la Lengua Extranjera, inglés). No obstante, los contenidos que se trabajan no abordan el bienestar docente como aspecto fundamental para la calidad de la educación.

El marco teórico de nuestra investigación se fundamenta en los estudios sobre el bienestar del profesorado y del profesorado en formación con especial hincapié en el profesorado de lenguas. Los docentes han sufrido durante las últimas dos décadas caídas significativas en sus niveles de bienestar personal y profesional. Por esta razón, los estudios de malestar y burnout se multiplicaron en todo el mundo, desde los años 80, para medir este fenómeno.

Nuestra investigación mostrará datos sobre un aspecto poco explorado del bienestar docente y cómo es percibido por el profesorado en formación; elemento que resultará de vital importancia para su futuro desarrollo personal y profesional. Este trabajo nos proporcionará los datos necesarios para reclamar una reorientación de los planes de estudio con el fin de que estos incluyan contenidos transversales de bienestar docente, sobre todo durante las prácticas externas puesto que la formación en este aspecto aumentará la motivación intrínseca de los docentes y, como consecuencia, elevará los estándares de una educación integral y de calidad.

\section{Preguntas de investigación}

Con este estudio pretendemos dar respuesta a las siguientes preguntas que guiarán el desarrollo conceptual y metodológico del mismo:

1. ¿Qué elementos percibe el profesorado de LE en formación como causantes de malestar docente?

2. ¿Qué elementos percibe el profesorado de LE en formación como causantes de bienestar docente?

3. ¿Cómo cree el profesorado de LE en formación que se podría mejorar el bienestar del docente de LE? 
Manuel J. Cardoso y Juan R. Guijarro Percepciones sobre el Bienestar Docente del profesorado...

\section{Marco teórico}

El marco teórico que vamos a presentar a continuación recoge los principales avances de lo que significa el bienestar docente para el profesorado en ejercicio y en formación y avalará la consecuente planificación metodológica de este estudio.

\subsection{El bienestar docente}

Aelterman et al. (2007) definen qué es el bienestar docente o teachers' wellbeing:

Well-being expresses a positive emotional state, which is the result of harmony between the sum of specific environmental factors on the one hand and the personal needs and expectations of teachers on the other (p. 2).

Esta definición se encuentra entre las de mayor aceptación internacional y su principal logro consiste en transformar la tendencia de elaborar estudios basados en el malestar docente, o burnout, a desarrollar las primeras nociones con objetivo positivo, como sería el bienestar o wellbeing.

Diversos estudios (Hoy y Miskel, 1996; Huberman y Vanderberghe, 1999; Smylie, 1999; Halliger, 2003) sostienen que los factores que afectan al bienestar personal de los docentes están directamente vinculados con el lugar de trabajo aunque también han indicado que el bienestar (o malestar) es una sensación subjetiva e individual. Aelterman et al. (2007) indican algunos factores que influyen en la sensación de bienestar docente que no forman parte del contexto profesional, como la personalidad o el bagaje personal, que es capaz de incidir tanto en la forma en la que los docentes lidian con las demandas sociales como en la propia praxis. Como resultado principal en su investigación, declaran que el respaldo o apoyo social en el lugar de trabajo es uno de los factores más importantes con el que hacer frente a situaciones que perturban el bienestar profesional y/o personal. De la misma manera, desarrollan un modelo de bienestar docente formado por tres categorías: aspectos personales, profesionales y sociales.

Horn, Tarris, Schaufeli y Schreurs (2004) se centran en los aspectos positivos de la docencia. Para ellos el bienestar ocupacional es una evaluación positiva de varios aspectos en la vida profesional individual. Esta investigación describe un modelo compuesto por cinco dimensiones:

1. Bienestar afectivo: Esta dimensión presenta aspectos negativos de la profesión como el agotamiento emocional- pero también aspectos positivos -satisfacción en el lugar de trabajo y compromiso organizacional-. El agotamiento emocional está relacionado con la interacción de los individuos con el resto del personal como, por ejemplo, los alumnos (Maslach, 1993). Asimismo, el compromiso organizacional hace referencia a la participación docente en el centro educativo.

2. Bienestar profesional: El bienestar profesional está relacionado con la aspiración, la competencia profesional y la autonomía. Esta última es considerada de vital 
importancia para los docentes ya que estiman que poseen bastante autonomía y poder en la escuela. No obstante, la autonomía suele limitarse al espacio del aula por lo que si surge un problema fuera de ella los docentes tienden a sentirse incompetentes, débiles y despreciados, en especial, cuando deben seguir unas normas impuestas por el equipo de gobierno.

3. Bienestar social: Esta dimensión manifiesta cuales son los comportamientos correctos del docente hacia sus alumnos y compañeros. Igualmente, el fomento del bienestar social provoca que disminuyan los niveles de despersonalización (base del malestar docente) y que aumente el interés, el afecto y la pasión en el lugar de trabajo.

4. Bienestar cognitivo: El bienestar cognitivo abarca aspectos propios del burnout pues explica que los docentes deben estar concentrados en su trabajo y, al mismo tiempo, enfrentarse de manera adecuada a nuevas situaciones.

5. Bienestar psicosomático: Esta dimensión contempla la presencia o ausencia de afecciones y síntomas psicosomáticos como, por ejemplo, dolores de cabeza y/o dolores de espalda. Según recogen los autores, los orígenes de las angustias y aflicciones psicosomáticas de los docentes se cimientan en los ambientes negativos de trabajo, tales como contextos altamente exigentes o con poca autoridad (Travers y Cooper, 1993)

Horn et al. (2004) afirman que el bienestar y la reducción del estrés en el lugar de trabajo -en nuestro caso, la escuela- se intensifican a través de actos positivos, motivaciones, conductas, percepciones y ejercicio físico. Asimismo, explicaron que los bajos niveles de bienestar se manifiestan de diversas formas, desde el cansancio extremo y el bajo compromiso laboral hasta la falta de concentración y afecciones psicosomáticas.

\subsection{Fuentes de malestar en docentes de LE}

A continuación, vamos a presentar varios estudios experimentales centrados en conocer las fuentes del malestar de los docentes de LE. En primer lugar, destaca una investigación realizada en Inglaterra y en Francia con 800 docentes de LE. Es conveniente resaltar que en Reino Unido cerca del $22 \%$ de los docentes de LE experimenta depresión mientras que en el país francófono solamente está deprimido el 1\% de los docentes. De la misma manera, el 55\% de los docentes anglosajones contempla abandonar la profesión pues consideran que están expuestos a fuertes presiones, frente al $20 \%$ de los docentes franceses. Además, este grupo de docentes cita diversos factores que les producen malestar como la disciplina del aula, la baja posición social de la asignatura y de la profesión, la falta de apoyo por parte de los padres, el aumento de las horas de trabajo y las interferencias políticas (Travers y Cooper, 1996).

En segundo lugar, Wieczorek (2016) lidera una investigación cualitativa en Polonia con el fin de identificar cuales son los factores que causan malestar a los docentes de LE. En este estudio, participaron veinticinco docentes de LE sin discriminar el tipo de centro del que provenían (primario, secundario, universitario, público y/o privado). Los resultados principales revelan dos categorías claramente diferenciadas. Dentro de la primera categoría 
se presentan los factores que provocan malestar docente sin discriminar el tipo de asignatura que se imparta, como: la falta de motivación y la actitud de los alumnos, los bajos resultados académicos, la presión social, el contexto escolar, la presión por el tiempo disponible, la sobrecarga laboral y la burocracia. De acuerdo con este estudio, la segunda categoría recoge entonces los factores específicos que, según los docentes polacos, modifican el bienestar en el aula de lengua extranjera: los grupos heterogéneos de alumnos donde es difícil atender las diferencias individuales; la falta de recursos y materiales de calidad y adecuados; la enseñanza de la competencia oral tanto en la recepción como en la producción de una LE; la enseñanza de la gramática para la que no disponen de tiempo por las grandes diferencias existentes entre el polaco y el inglés; la implementación de nuevos materiales para los que no reciben formación suficiente.

Como se puede inferir, esta investigación revela que los factores descritos -a excepción de los grupos heterogéneos-, son el resultado de las inhibiciones individuales y de los sentimientos de baja autoestima hacia el nivel de competencia lingüística personal. Las conclusiones derivadas son por tanto, que los docentes de LE se sienten estresados debido a sus propias inseguridades. Asimismo, indican que les estresa tener lagunas de vocabulario, cometer errores gramaticales, tener problemas de pronunciación y el hecho de tener que hablar la LE durante todo el día. No obstante, Wieczorek (2016) aclara que muchos de estos factores no son verdad, puesto que no se han observado casos de malas prácticas en LE, sino que son miedos causados por una autoestima baja y por la actitud que tiene la sociedad actualmente (padres, alumnos y compañeros) hacia la asignatura de LE y la profesión docente.

\subsection{El bienestar docente dentro de la formación inicial}

Borg (2006) lideró una investigación de carácter cualitativo-narrativo para esclarecer la percepción de los docentes de LE sobre su profesión y averiguar qué elementos podrían causar un descenso en su bienestar docente.

En lo relativo a métodos, actividades y materiales, se ve como positivo el hecho de que los docentes de LE disponen de más materiales y más dinámicos que el resto de docentes (juegos, canciones, audiovisuales, etc.). En relación al contenido que se enseña, dado que en numerosas ocasiones implica enseñar la cultura que hay detrás de la lengua y todas sus esferas (educación, política, lingüística, historia...), se retrata también como elemento de fomento del bienestar precisamente por ese nivel de diversidad temática y actual, cercana al alumno. Otro ámbito de exploración positiva es la naturaleza del lenguaje. Vinculado a esta categoría, los docentes en formación mencionaron que la enseñanza implica tener un vasto conocimiento de la lengua y, además, mantenerse actualizado en lo que respecta al uso de nuevos términos y la formación de nuevas palabras. Indicaron asimismo, que la LE difiere del resto de asignaturas en que representa importancia práctica de forma inmediata y a largo plazo.

Cuando se refieren a la relación con los alumnos, las relaciones sociales con los alumnos suelen ser más cercanas, más relajadas y generalmente más positivas que con el resto de docentes. Los docentes en formación citan que esta característica se debe, posiblemente, a que los alumnos tienden a contar sus propias experiencias y sus vidas, lo que crea ambientes positivos de trabajo que, según ellos, son más humanos y agradables. 
Un área problemática viene del hecho de ser nativo de la LE o no. Los docentes de lenguas se deben concentrar más en lo que están explicando ya que también deben estar atentos a aspectos fonológicos, por ejemplo. Por esta razón, los docentes en formación estipulan que los docentes de LE pasan más tiempo preparando sus clases ya que tienen que pensar detenidamente cómo van a explicar los conceptos usando la LE para que todos los alumnos puedan aprender y captar la idea principal. Esta situación suele ocupar un puesto relevante como elemento que disminuye el bienestar.

Por último, en lo concerniente a las características personales, los participantes presentaron una lista con características deseables en los docentes de LE, destacando: que tenga sentido del humor, que sea creativo, flexible, motivador, simpático e irradie sentimientos positivos. Todos estos elementos suponen una constante demanda por parte del profesorado de LE y tiene esa doble vertiente de motivación pero también de elemento estresante. No obstante, más tarde acordaron que esta lista era esencial en los docentes de LE pero que también era deseable en el resto de docentes.

El trabajo de González, Montoya y Sierra (2002), recopila una serie de narrativas con maestros de inglés colombianos a través de grupos focales y cuestionarios. La idea básica que deseaban desarrollar residía en romper con el enfoque escolar tradicional para pasar a un enfoque integral. González y su equipo indican que el enfoque integral aboga por un docente de lenguas con tres roles: instructor, trabajador y aprendiz. Los docentes de LE consideran que las siguientes características ayudarían a desarrollar su bienestar docente y personal:

a) El docente como trabajador: mejores salarios; menor sobrecarga de trabajo adicional como, por ejemplo, los trámites burocráticos; un trabajo estable ya que la mayoría de los profesores de inglés no tienen una plaza fija; autonomía para tomar decisiones ya que la administración de la escuela impone sus opiniones con respecto a la selección de libros de texto, la frecuencia de horas de inglés a la semana y los recursos materiales disponibles; prevención de la violencia en las aulas olvidada por el gobierno; creación de un espacio para compartir, desarrollar y discutir ideas.

b) El docente como instructor: menor número de estudiantes por clase para el óptimo desarrollo de la competencia comunicativa; mayores recursos materiales, audiovisuales, TIC, etc.; más clases de inglés a la semana que mejorarían la competencia del alumnado así como su motivación hacia la LE; uso de enfoques transversales multidisciplinarios creando un clima de tolerancia y globalización; profundización en las técnicas didácticas del inglés para un mejor desempeño profesional.

c) El docente como aprendiz: mejora de la competencia lingüística del profesorado; instauración de una red de contactos interdisciplinarios para solventar problemas de hiperactividad, nutrición, etc.; aumento de la formación continua académica del profesorado; promoción de la enseñanza reflexiva y enfoques humanísticos con el fin de educar a los alumnos holísticamente.

González et al., (2002) explican que los docentes de inglés como LE en Latinoamérica necesitan estrategias que mejoren su praxis a través del conocimiento de sus roles como trabajadores, como instructores y como aprendices. No obstante, los programas que se ofertan 
Manuel J. Cardoso y Juan R. Guijarro Percepciones sobre el Bienestar Docente del profesorado...

no suelen ser conscientes de las necesidades de los docentes como aprendices, por lo que no pueden desarrollar ni su autonomía ni su metodología. De realizarse correctamente, bajaría la sensación de burnout y aumentaría exponencialmente la de bienestar.

\section{Metodología}

\subsection{Contexto}

Nuestro estudio se lleva a cabo en la Facultad de Ciencias de la Educación de la Universidad de Granada. Los integrantes de esta investigación son estudiantes del Grado en Educación Primaria que han decidido especializarse en Lengua Extranjera (inglés). Para la obtención de esta especialidad los estudiantes deben cursar, durante el primer semestre del último curso, cuatro asignaturas centradas en las didácticas específicas de la lengua extranjera (inglés): Enseñanza y aprendizaje de la LE; competencia comunicativa de la LE; Didáctica de la cultura de la LE; y Didáctica de la ficcionalidad de la LE. Asimismo, los estudiantes deben realizar sus prácticas externas como docentes de LE durante 15 semanas en colegios del área urbana y metropolitana de Granada.

\subsection{Participantes}

Todos los participantes cursaban durante el transcurso de la investigación la materia de Prácticum II (240 horas en aula) la cual se localiza en el octavo semestre de su formación inicial. Para poder acceder a esta materia los participantes debían superar con anterioridad el Prácticum I y, como mínimo, 160 ECTS de los 240 totales del plan de estudios. El número de participantes fue de 12 personas, 4 mujeres y 8 hombres, todos hablantes nativos de español y con un conocimiento acreditado de la lengua inglesa de nivel B2.

Tabla 1. Información sobre los participantes del grupo de discusión

\begin{tabular}{|c|c|c|}
\hline Participante & Género & Edad \\
\hline P1 & Hombre & 22 \\
\hline P2 & Mujer & 23 \\
\hline P3 & Hombre & 21 \\
\hline P4 & Mujer & 22 \\
\hline P5 & Hombre & 24 \\
\hline P6 & Mujer & 23 \\
\hline P7 & Hombre & 27 \\
\hline P8 & Mujer & 22 \\
\hline P9 & Hombre & 23 \\
\hline P10 & Hombre & 22 \\
\hline P11 & Hombre & 22 \\
\hline P12 & Hombre & 22 \\
\hline
\end{tabular}




\subsection{Instrumento}

Como instrumento de investigación hemos desarrollado un grupo de discusión porque nos permite profundizar en los aspectos del bienestar docente de manera dialógica dentro de la formación inicial. En el mecanismo propio de un grupo de discusión se pueden confrontar ideas $\mathrm{y}$, de esa manera, los datos proporcionados por cada participante quedan desarrollados de manera compleja y argumentada.

Nuestro instrumento consta de una serie de preguntas relacionas con las vidas personales y profesionales de los informantes que fueron evaluadas y perfiladas por tres jueces expertos en la materia externos a la Universidad de Granada. Asimismo, estos interrogantes no admiten una respuesta dicotómica sino que se trata de responder a las preguntas argumentando sus creencias y, sobre todo, experiencias personales relacionadas con el bienestar docente.

Por un lado, las preguntas que les lanzábamos les hacían reflexionar sobre cómo ve el resto de la sociedad su trabajo como docentes y si esta visión repercute en sus niveles de bienestar. Por otro lado, les pedíamos que narraran cómo habían superado una situación difícil dentro del aula y qué sentimientos (de bienestar o malestar) experimentaron cuando se enfrentaron a estos retos así como a la hora de adaptarse a la mecánica del centro escolar. De la misma manera, les preguntamos qué acciones o situaciones elevaban sus niveles de autoestima y bienestar personal y/o profesional. Más tarde, les pedimos que enumeraran una serie de cualidades deseables en un buen docente de lengua extranjera y en qué grado se sentían motivados para alcanzarlas. Finalmente, les preguntamos si habían sido capaces de trasladar los conceptos entrenados en las diferentes asignaturas durante su formación universitaria al aula de educación primaria.

\subsection{Procedimiento}

Durante el segundo semestre del año 2015 se elaboraron las preguntas del grupo de discusión. A partir de ahí razonamos que la mejor fecha para conducir nuestro grupo de discusión era a partir de la segunda quincena de abril de 2016 dado que los estudiantes ya habían pasado el ecuador de sus prácticas externas y podían expresarse con más feedback que si dirigiésemos el grupo de discusión a comienzos del Prácticum II en febrero de 2016.

Los datos personales de los participantes son completamente anónimos con el fin de preservar la identidad de los mismos. El grupo de discusión se llevó a cabo en un espacio facilitado por la Facultad de Ciencias de la Educación y duró más de dos horas y media. La recogida de datos se llevó a cabo con soportes de almacenamiento digital en video. Consideramos oportuno el uso de videograbadoras con el fin de analizar tanto el lenguaje oral producido como la kinésica de los informantes.

La primera parte de la sesión constó de una presentación sobre aspectos clave de lo que científicamente se entiende por bienestar docente donde los participantes realizaron preguntas para aclarar conceptos. Seguidamente, los investigadores empiezan a lanzar las preguntas que se han diseñado. Tras unas preguntas iniciales para romper el hielo y crear un clima relajado de confianza entre la cohorte, se empieza la sesión propiamente dicha. Una vez finalizamos este proceso de investigación transcribimos todo el contenido para su posterior análisis. 
Manuel J. Cardoso y Juan R. Guijarro Percepciones sobre el Bienestar Docente del profesorado...

\subsection{Análisis de datos}

Siguiendo las orientaciones metodológicas de Miles, Huberman y Saldaña (2014), los datos obtenidos han sido analizados por los dos investigadores de este trabajo a través de tres lecturas profundas de las transcripciones en intervalos de diez días. Este procedimiento nos ha permitido codificar categorías temáticas con un grado de objetividad alto y considerar todos los matices posibles al contrastar los dos puntos de vista de los investigadores. Hemos pretendido siempre comprender las experiencias de todos los informantes, los puntos comunes y los individuales. Este análisis comparativo ha permitido diseccionar los datos en partes y codificarlos en temas emergentes a través de este proceso iterativo.

\section{RESUltados y ANÁlisis}

A continuación, vamos a exponer los resultados más sobresalientes de nuestro grupo de discusión e ilustrar con ejemplos las opiniones de los informantes en base a las preguntas de investigación planteadas al inicio de este trabajo:

1. ¿Qué elementos percibe el profesorado de LE en formación como causantes de malestar docente?

En primer lugar, la práctica totalidad de participantes declaran que el elemento que mayor malestar docente les ocasiona una vez que tienen que afrontar las prácticas externas es una serie de carencias formativas durante el Grado en Educación Primaria, así como también de la mención de LE. Coincide este elemento con los datos aportados por Wieczoreck (2016) en el contexto polaco.

Entre las diversas cuestiones señaladas, destacan la falta de formación en metodologías cooperativas que, una vez en los colegios, perciben como de vital importancia. Lo vemos ilustrado en este comentario de P12:

P12: "los aprendizajes cooperativos tienen un beneficio social porque [los alumnos y profesores] están aprendiendo a colaborar en equipos y no encerrarse solamente en su trabajo".

Asimismo, los participantes indican que les genera malestar el desconocimiento del funcionamiento interno de la profesión docente en los centros y que este conocimiento no se les ha proporcionado tampoco en la universidad:

P7: "la universidad debería estar mucho más cerca del colegio, me parece como cosa independiente, que aquí se forman teóricos y allí [en los colegios] trabajan los prácticos y cuando llegas no sabes adaptarte bien a la situación".

Los participantes afirman, igualmente, que las asignaturas del Grado no ofrecen suficientes supuestos prácticos y esto les genera malestar a la hora de llevar a cabo su praxis docente: 
P5: "Yo una situación en el que tú me dices en el que leo en un folio que un niño tiene un problema y hay que hacerle una adaptación curricular, yo en el examen te voy a poner que a ese niño hay que hacerle una adaptación curricular, pero luego a la hora de estar en clase tendría que consultar informes o cualquier cosa para saber qué hacer con ese niño".

P11: “por ejemplo los de Matemáticas que tú te pones a contar con el ábaco o a hacer actividades con tangram y cosas de esas, no es lo mismo que yo lo haga aquí que hacerlo con niños, o sea no tienes el mismo ambiente, las mismas voces, las mismas formas de pensar que tienen ellos, entonces es algo diferente, tú puedes aplicar lo que tú sabes de hacerlo [conocimientos propios] pero no el cómo lo harían ellos".

Igualmente, todos los informantes se mostraron de acuerdo con P11 cuando declaró que la falta de formación práctica en la Universidad hace que se sientan perdidos en los centros escolares cuando se enfrentan a situaciones nuevas. Lo peor en este caso es que echan mano de los modelos aprendidos de sus anteriores profesores y reconocen que no siempre fueron los mejores posibles:

P11: "el no tener aquí unas referencias prácticas lo que te hace es tirar de tus recuerdos de cómo te dieron a ti las clases tus profesores e intentas darlas o intentas imitar lo que hacían tus profesores y nos hemos dado cuenta de que muchos no tenían razón, no lo hacían bien, y ahora estamos copiándolo porque no tenemos otra referencia nueva".

P5: "[mi paso por el sistema educativo] siempre ha sido todo muy competitivo",

P8: "[ahora como docente me genera malestar que los alumnos] no te hacen ni caso, o sea les puedes decir lo que sea, les da igual todo".

La primera afirmación corrobora el estudio que llevaron a cabo Calderhed y Robson (1991) en el que describieron cómo los docentes en formación recuerdan con bastante precisión su paso por el sistema educativo. Vinculado a esto, algunos informantes admitieron que les gustaría cambiar los aspectos negativos que recuerdan tras su paso por la escuela primaria con el fin de desarrollar futuras metodologías eficientes, lo que concuerda con el estudio de Gupta y Saravanan (1995). Además, el último aspecto comentado por P8 coincide con el agotamiento emocional descrito en Maslach (1993) pues reconoció que sus niveles de bienestar se veían afectados dependiendo de la calidad de sus relaciones con los alumnos y alumnas.

Los participantes también afirmaron que se sentían cohibidos porque no llevaban el control total de la clase y/o no concordaban con la metodología de su tutor del centro. Este sentimiento está íntimamente relacionado con el hecho de que 8 participantes señalaron que a pesar de que sus centros eran bilingües, los maestros de inglés no hablaban en inglés: 
Manuel J. Cardoso y Juan R. Guijarro Percepciones sobre el Bienestar Docente del profesorado...

P3: "el speaking no se utiliza en nuestro colegio bilingüe. Mi tutor me ha dicho que [utilizar el inglés] es una tarea pendiente. Tiene miedo a que no le entiendan".

Como último elemento generador de malestar, los participantes comentaron que experimentaban sentimientos de desánimo debido a que la sociedad no valoraba su profesión:

P4: "los amigos, y todo el mundo, te están diciendo que eres maestro y que no haces falta, como que no eres lo mismo que un médico";

P7: "me da un poco de impotencia porque creo que [la sociedad] no valora lo que es la educación, lo importante que es, me da un poco de rabia que sea gente joven la que lo dice";

P5: "mi madre quería que fuese ingeniero químico y que hiciera la carrera de ingeniería química, porque un ingeniero está mejor posicionado. Para mi madre estudiar magisterio era un tiempo perdido [...] mi madre luego sí que me apoya pero me dijo: te vas a meter ahí si eso son cuatro años estudiar, luego no sabes dónde te vas... mejor estudia química".

Estas afirmaciones coinciden con el estudio de Aelterman et al. (2007) pues los participantes también declararon que el respaldo y el apoyo social es importante durante la praxis docente e influye en sus niveles de bienestar. No obstante, algunos informantes aclararon que sus familiares les apoyan y les ayudan, sobre todo aquellas familias con ascendientes docentes.

2. ¿Qué elementos percibe el profesorado de LE en formación como causantes de bienestar docente?

En primer lugar y como elemento indiscutible, todos los participantes expresaron o asintieron que durante el periodo de prácticas externas sus niveles de autoestima y bienestar se elevaban cuando sus alumnos mostraban signos de aprendizaje como la manera perfecta de sentirse útil en su campo profesional. P4 lo expresa así:

P4: "me gusta sentirme útil [...] enseñas a un niño palabras nuevas en inglés y que el niño las aprenda, a mí eso me satisface personalmente".

Los participantes señalan como elementos de bienestar esa aspiración profesional y personal de los docentes en formación de convertirse en buenos docentes. La mayoría de los informantes expresaron que todos los docentes deberían aspirar a compartir una serie de cualidades positivas generales para sentirse mejor como:

P3: "que trabaje con aprendizaje cooperativo y sea motivador";

P6: "ser un profesor dinámico y hacer las clases interactivas". 
Como podemos observar, algunas de estas cualidades coinciden con las investigaciones de Weinstein (1988) y Borg (2006) cuando definen que el 'buen' docente es aquel que es empático y motivador.

Tras un extenso debate, los participantes concluyeron que los docentes de LE se sitúan en un lugar único y privilegiado dentro del prisma educativo, lo que es un elemento motivador positivo, e indicaron asimismo qué cualidades los diferencian del resto de docentes:

P10: "conocimiento del idioma";

P12: "saber comunicarse en inglés y hacer las sesiones de acuerdo al inglés que vamos a tener en un futuro";

P5: "la creatividad es un punto muy a favor de un profesor de inglés ya que tiene que relacionar los conceptos de todas las otras asignaturas comunes, porque en inglés se dan animales que se da en conocimiento del medio, se dan los números que son de matemáticas, se dan estructuras gramaticales que es de lengua".

Aquí recogemos el punto de vista de P12, que coincide con el resto de la cohorte, sobre la necesidad del incremento de la valoración social de la profesión del docente de Primaria como pilar importante en el aumento de su bienestar:

P12: "otra cosa que si me gustaría es que se valorase más la profesión".

En último lugar, los informantes expresaron como aspectos de bienestar de su desempeño docente: el sentimiento del trabajo bien hecho, la preocupación positiva por los alumnos, la atención a la diversidad y el ayudar a los niños a desarrollarse integralmente:

P5: “ $[\ldots]$ maestros buenos que se preocupen por los niños, que se valoren las diferentes inteligencias, las diferentes capacidades, que hay muchos niños que tienen muchas capacidades para hacer cosas, es necesario que haya un buen bailarín, un buen pintor o buen escultor";

Estos últimos aspectos ponen de manifiesto los deseos de los participantes en lo que respecta a una restructuración de las materias del Grado para que estas incluyan aspectos transversales como el aprendizaje y uso de las inteligencias múltiples. De la misma manera, observamos que los participantes manifestaron una predisposición positiva hacia la profesión docente.

\section{3. ¿Cómo cree el profesorado de LE en formación que se podría mejorar el bienestar del docente de LE?}

Para esta cohorte es fundamental que se fomente el clima positivo de aprendizaje en el aula y se lidie de manera efectiva con los problemas emocionales del alumnado. Vemos cómo lo expresan P2, P5 y P10: 
Manuel J. Cardoso y Juan R. Guijarro Percepciones sobre el Bienestar Docente del profesorado...

P2: "es necesario que los niños sepan de los problemas de los demás. Los alumnos no tienen ese sentimiento desarrollado de decir: 'vamos a ponernos en su piel por un momento'. Si estamos en cualquier clase esto [los problemas personales de los alumnos] yo creo que es más importante que cualquier asignatura, si el clima del aula no está yendo bien, los compañeros no se entienden entre ellos, si la clase no va bien no podemos seguir una asignatura, ellos [los alumnos] tienen que tener en cuenta que están trabajando como grupo no como personas sueltas";

P5: "que el docente valore [al alumno] más allá de lo que sabe en un examen";

P10: "hay que ser positivo y favorecer la motivación".

Como podemos observar, los participantes concuerdan con las afirmaciones de Book, Byers y Freeman (1983) cuando indican que es más importante enseñar a los alumnos a manejar sus preocupaciones que contenidos formales. Además, describen que en las aulas no se afrontan los problemas emocionales. Más concretamente, observamos cómo los participantes ponen de relieve que estas cualidades no se localizan en las aulas debido a que la formación inicial no profundiza en las dimensiones sociales y emocionales propias de la profesión docente,

P7: "es importante que el centro utilice la misma metodología, todas las asignaturas $\mathrm{y}$ todos los maestros en todos los cursos hagan lo mismo, no unos los trabajos cooperativos y [otros docentes hagan] otra cosa diferente";

P11: "los grupos deberían ser mixtos, conociendo a los alumnos según cómo son de personalidad, de que haya variedad para que cada grupo tenga diferentes puntos de opinión”.

Si analizamos los comentarios de P7, observamos que está en la línea de la principal hipótesis de Horn et al. (2004) cuando señala que todos los miembros del centro escolar deberían trabajar en equipo con el fin de impulsar las buenas prácticas y el bienestar docente. Sin embargo, manifiesta que no es posible dado que durante la formación inicial no se fomentan efectivamente las habilidades interpersonales necesarias para trabajar en grupo y favorecer los ambientes de aprendizaje positivos. P11 manifiesta que la técnica de la creación de grupos heterogéneos debería estar más extendida en los centros escolares ya que, según ellos, motiva al alumnado y refuerza las relaciones sociales. Sin embargo, el plan de estudios es estéril en este sentido.

\section{IMPLICACIONES DIDÁCTICAS-FORMATIVAS}

De acuerdo con los resultados que han aflorado, se pone de manifiesto que la formación inicial de los docentes, en especial la de los docentes de LE, necesita un (re)ajuste en lo 
que respecta a contenidos formales en materia de bienestar docente. Tal y como se puntualiza en el grupo de discusión, la formación académica no contiene el suficiente número de supuestos prácticos que fomenten el conocimiento total de la profesión y este aspecto les genera sentimientos de malestar cuando acuden al aula. Igualmente, estos estudiantes indican que no poseen una formación sólida en bienestar docente, en estrecha relación con las emociones y las inteligencias múltiples, por lo que tienen que recurrir a sus experiencias pasadas como alumnos ya que no conocen otra forma de enseñar. Esta afirmación supone el reconocimiento de que no existen contenidos en los planes de estudio que desarrollen integralmente el bienestar docente como elemento multifactorial desde la didáctica general y la específica.

A nuestro juicio, creemos que es necesario escuchar atentamente las voces de nuestro profesorado en formación y promover que materias curriculares fundamentales como el Prácticum I y II reajusten e incorporen de manera transversal las cuestiones que han aflorado sobre el bienestar docente.

\section{Conclusiones}

Para finalizar nuestro estudio vamos a ver de manera global en qué forma han quedado contestadas las preguntas de investigación que planteábamos al inicio de la investigación.

En lo que respecta a los elementos que se perciben como causantes del malestar docente, hay que destacar las carencias formativas en la formación ofrecida por la Universidad como es la falta de formación con casos prácticos; el desconocimiento del funcionamiento interno de los centros; la falta de valoración social de la profesión y el observar cómo en centros bilingües los maestros en ejercicio no se comunican en inglés con su alumnado.

En referencia a los aspectos que marcan y fomentan el bienestar docente, queda explicitado que los relevantes para nuestro grupo son el percibir cómo los alumnos aprenden y aplican lo que ellos les enseñan; la motivación de convertirse en buenos docentes de LE, ya que consideran que son privilegiados respecto a otras especialidades; el sentimiento del trabajo bien hecho, la preocupación positiva por los alumnos, la atención a la diversidad y el ayudar a los niños a desarrollarse integralmente.

Las propuestas que hace nuestro profesorado de LE en formación para mejorar el bienestar docente vienen de la mano de la necesidad de entrenar al profesorado para el trabajo en equipo como vía para impulsar las buenas prácticas y el bienestar docente. Para esta cohorte es fundamental que se fomente el clima positivo de aprendizaje en el aula y se lidie de manera efectiva con los problemas emocionales del alumnado.

Contestadas las preguntas de investigación iniciales, cabe destacar en último lugar la necesidad imperiosa de la mejora del plan de formación de docentes de Primaria que está en curso en la actualidad para adecuarlo, principalmente, a las necesidades reales del sistema educativo. Paralela a esto, se manifiesta la necesidad de que facultades de educación y centros educativos deberían trabajar de manera coordinada para ofrecer a los futuros docentes una formación integral de calidad. 
Manuel J. Cardoso y Juan R. Guijarro Percepciones sobre el Bienestar Docente del profesorado...

\section{Referencias}

Aelterman, A., Engels, N., Van Petegem, K. y Pierre Verhaeghe, J. (2007). "The well-being of teachers in Flanders: the importance of a supportive school culture", en Educational Studies, 37, 3: 285-297.

Book, C., Byers, J. y Freeman, D. (1983). "Student expectations and teacher education traditions with which we can and cannot live", en Journal of Teacher Education, 34, 1: 9-13.

Borg, S. (2006). "The distinctive characteristics of foreign language teachers", en Language Teaching Research, 10, 1: 3-31.

Calderhead, J. y Robson, M. (1991). "Images of teaching: Student teachers' early conceptions of classroom practice", en Teaching and Teacher Education, 7: 1-8.

González, A., Montoya, C. y Sierra, N. (2002). "What do EFL teachers seek in Professional Development Programs? Voices from the teachers", en Íkala. Revista de Lenguaje y Cultura, 7: 29-50.

Gupta, R. y Saravanan, V. (1995). "Old beliefs impede student teacher learning of reading instruction", en Journal of Education for Teaching, 21: 347-360.

Hallinger, P. (2003). "Leading educational change: reflections on the practice of instructional and transformational leadership", en Cambridge Journal of Education, 33: 329-351.

Horn, J. E. van, Taris, T. W., Schaufeli, W. B. and Shreurs, P.J.C. (2002): "A multi-dimensional approach to measuring teacher well-being". Capítulo 3. Presentado para publicar, 64-81.

Hoy, W.K. y Miskel, C.G. (1996). Educational administration: Theory, research and practice. New York: McGraw-Hill.

Krueger, R. A. (1991). El grupo de discusión. Guía práctica para la investigación aplicada: Madrid, Pirámide.

Kyriacou, C. (2001). "Teacher stress: Directions for future research", en Educational Review, 53, 1: 27-35.

Maslach (1993). "Burnout: A multidimensional perspective", en Schaufeli, W. B., Maslach, C. y Marek, T. (eds), Professional burnout: Recent Developments in Theory and Research. Philadelphia: Taylor \& Francis, 19-32.

Miles, M.B.; Huberman, A.M., y Saldaña, J. (2014). "Fundamentals of qualitative data analysis", en M.B. Miles, A.M. Huberman y J. Saldaña (eds.). Qualitative data analysis: A methods sourcebook. Los Angeles, CA: Sage, 69-104.

Pérez Valverde, C. y Ruiz Cecilia, R. (2014). "Narrativas de la identidad docente en la formación del profesorado de lenguas extranjeras", en Andamios, 11, 24: 215-234.

Smylie, M.A. (1999). "Teacher stress in a time of reform", en R. Vandenberghe y A.M. Huberman (eds.). Understanding and preventing teacher burnout. Cambridge: Cambridge University Press.

Travers, C. J. y Cooper, C. L. (1996). Teachers under pressure: Stress in the teaching profession. London: Routledge.

Van Horn, J.E., Taris, T.W., Schaufeli, W.B., y Shreurs, P. J. (2004). “A Multidimensional Approach to Measuring Teacher Wellbeing", en Journal of Occupational and Organisational Psychology, 77: 365-375.

Vandenberghe, R. y A. M. Huberman, (1999). Understanding and Preventing Teacher Burnout: A Source of International Research and Practice. Cambridge: Cambridge University Press.

Weinstein, C. (1988). "Preservice teachers' expectations about the first year of teaching", en Teaching and Teacher Education, 4, 1: 31-40. 
Wieczoreck, A. (2016): "High Inhibitions and Low Self-esteem as Factors Contributing to Foreign Language Teacher Stress", en D. Gabryś-Barker y D. Gałajda (ed.), Positive Psychology Perspectives on Foreign Language Learning and Teaching. Switzerland: Springer, 231-247. 